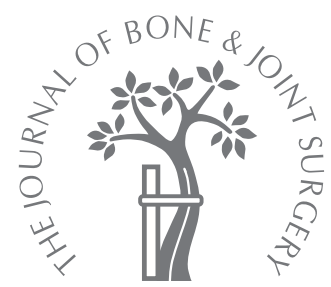

- SPINE

\title{
The Andersson lesion in ankylosing spondylitis
}

\author{
DISTINGUISHING BETWEEN THE INFLAMMATORY AND \\ TRAUMATIC SUBTYPES
}

\begin{abstract}
Y.-S. Park,
J.-H. Kim,

J.-A. Ryu,

T.-H. Kim
\end{abstract}

From Guri Hospital, Hanyang University College of Medicine, Kyunggi-do, Korea

Y.-S. Park, MD, Orthopaedic Surgeon, Professor

J.-H. Kim, MD, Orthopaedic Surgeon

Department of Orthopaedic Surgery

J.-A. Ryu, MD, Radiologist, Professor

Department of Radiology Guri Hospital, Hanyang

University College of Medicine, Gyomun 1-dong, Guri-si, Kyunggi-do, Korea.

n.-H. Kim, MD,

Rheumatologist, Professor Hanyang University Hospital for Rheumatic Disease,

Haengdang 1-dong,

Seongdong-gu, Seoul, Korea.

Correspondence should be sent to Professor Y.-S. Park; e-mail: hyparkys@ hanyang.ac.kr

(C)2011 British Editorial Society of Bone and Joint Surgery doi:10.1302/0301-620X.93B7. $26337 \$ 2.00$

$J$ Bone Joint Surg $[\mathrm{Br}]$ 2011;93-B:961-6.

Received 6 December 2010;

Accepted after revision 9 March 2011

A number of causes have been advanced to explain the destructive discovertebral (Andersson) lesions that occur in ankylosing spondylitis, and various treatments have been proposed, depending on the presumed cause. The purpose of this study was to identify the causes of these lesions by defining their clinical and radiological characteristics.

We retrospectively reviewed 622 patients with ankylosing spondylitis. In all, 33 patients $(5.3 \%)$ had these lesions, affecting 100 spinal segments. Inflammatory lesions were found in 91 segments of 24 patients $(3.9 \%)$ and traumatic lesions in nine segments of nine patients (1.4\%). The inflammatory lesions were associated with recent-onset disease; a low modified Stoke ankylosing spondylitis spine score (mSASSS) due to incomplete bony ankylosis between vertebral bodies; multiple lesions; inflammatory changes on MRI; reversal of the inflammatory changes and central bony ankylosis at follow-up; and a good response to antiinflammatory drugs. Traumatic lesions were associated with prolonged disease duration; a high mSASSS due to complete bony ankylosis between vertebral bodies; a previous history of trauma; single lesions; nonunion of fractures of the posterior column; acute kyphoscoliotic deformity with the lesion at the apex; instability, and the need for operative treatment due to that instability.

It is essential to distinguish between inflammatory and traumatic Andersson lesions, as the former respond to medical treatment whereas the latter require surgery.

The destructive lesions that occur in the vertebral bodies and intervertebral discs in ankylosing spondylitis were first described by Andersson in $1937 . .^{1}$ Since then, they have been termed Andersson lesions, spondylodiscitides, destructive vertebral lesions and spinal pseudarthroses. ${ }^{2-5}$ This uncertainty of designation is due to a lack of agreement on the cause and pathology of the lesions. Two main theories have been proposed. The first is that they are inflammatory in nature and part of the natural history of ankylosing spondylitis itself;; 5 and the other is that they are traumatic lesions, and as such constitute pseudarthroses after trauma. ${ }^{7-9}$ Previous studies have reported different radiological and clinical characteristics based on these two theories. ${ }^{10-12}$ In this paper we have sought to identify the causes of Andersson lesions in ankylosing spondylitis by defining their radiological and clinical characteristics.

\section{Patients and Methods}

We carried out a retrospective study of a cohort of 622 patients who had been diagnosed with ankylosing spondylitis according to the modified New York criteria ${ }^{13}$ between March 2003 and
June 2009. There were 597 men and 25 women, with a mean age at first visit of 23.4 years (16 to 66). Andersson lesions were classified as inflammatory or traumatic, depending upon the previously reported radiological features. The criteria for an inflammatory lesion were narrowing of the disc space, erosion of the end-plate, and abnormal radiodensity of the adjacent vertebral body but without severe destruction (Fig. 1a); $5,6,11,12$ while those for a traumatic lesion were sclerosis and hypertrophy of the bone adjacent to the disc with accompanying intervertebral disc destruction and severe osteolysis of the vertebral body (Fig. 1b)., ${ }^{7,11,12}$ The sites of the lesions, typical findings, and changes during follow-up were examined by plain radiography, CT and MRI. The modified Stoke ankylosing spondylitis spine score (mSASSS) ${ }^{14}$ was used to quantify spinal involvement, especially bony ankylosis. Patients were scored from 0 to 3 on plain radiographs at each of the 24 anterior corners of the vertebral bodies from C1 to L5. Based on a total possible score of 72, a score of 0 indicated normality and a score of 72 indicated complete bony ankylosis or 'bamboo spine'. 


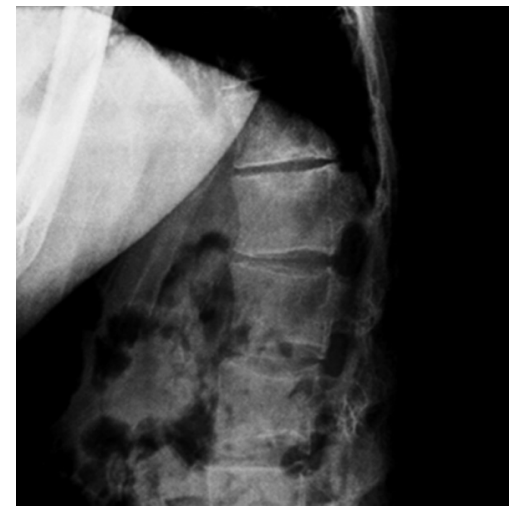

Fig. 1a

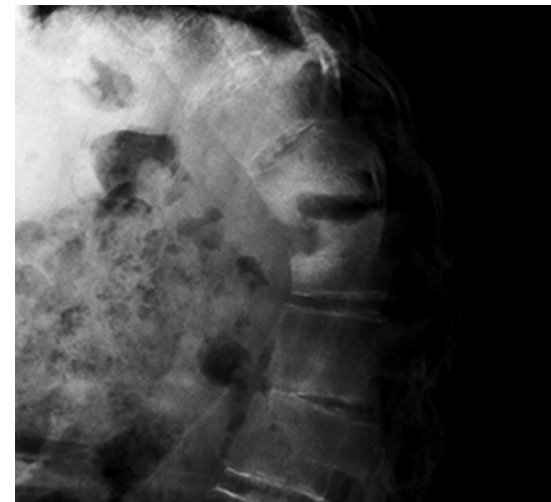

Fig. 1b

Radiographs showing a) an inflammatory Andersson lesion at T12-L1 with disc space narrowing, erosion of the endplate and abnormal radiodensity of the adjacent vertebral body but without severe destruction, and b) a traumatic Andersson lesion at T12-L1 with intervertebral disc destruction and severe osteolysis of the vertebral body.

Table I. Site of the 91 inflammatory Andersson lesions

\begin{tabular}{lc}
\hline Level & Cases $(\mathbf{n}, \mathbf{\%})$ \\
\hline T7-8 & $1(1.1)$ \\
T9-10 & $2(2.2)$ \\
T10-11 & $3(3.3)$ \\
T11-12 & $15(16.5)$ \\
T12-L1 & $19(20.9)$ \\
L1-2 & $16(17.6)$ \\
L2-3 & $7(7.7)$ \\
L3-4 & $8(8.8)$ \\
L4-5 & $9(9.9)$ \\
L5-S1 & $11(12.1)$ \\
\hline
\end{tabular}

\section{Results}

Andersson lesions were detected in 100 spinal segments in 33 patients $(5.3 \%)$.

Radiological findings. Characteristics of inflammatory lesions. Inflammatory lesions were identified in $91 \mathrm{seg}$ ments of 24 patients $(3.9 \%)$ (Table I). Single lesions were seen in four patients and multiple lesions in the remaining 20. The inflammatory lesions originated in the vertebral column in the presence of incomplete bony ankylosis, and were not accompanied by instability at the apex of the lesion, or by fracture of the posterior column.

The MRI signal intensity in the adjacent vertebral bodies varied according to the duration of the inflammation. In cases of acute inflammation, low signal intensity was seen on the $\mathrm{T}_{1}$-weighted MR images and high signal intensity on the $\mathrm{T}_{2}$-weighted images (Fig. 2a). In the chronic phase, the situation was reversed (Fig. 2b). The mean mSASSS in these 24 patients was 16.05 (10 to 25 ).

Characteristics of traumatic lesions. Traumatic lesions were found in nine segments of nine patients $(1.4 \%)$ (Table II) and in each case they were restricted to a single level. The ankylosed spine was severely disrupted by the lesion and there was an associated mean acute kyphotic deformity of
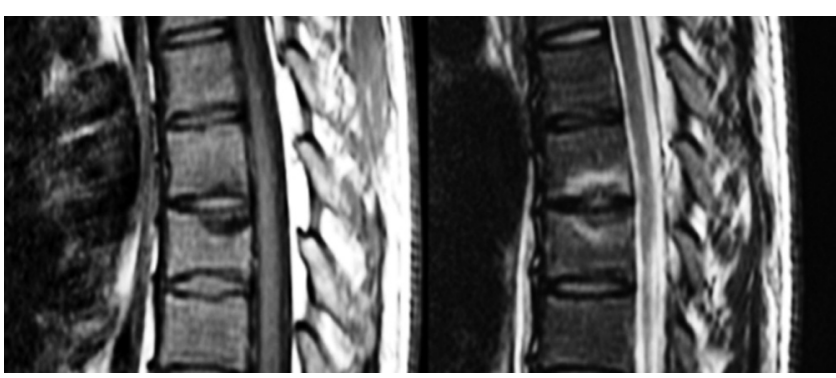

Fig. 2a
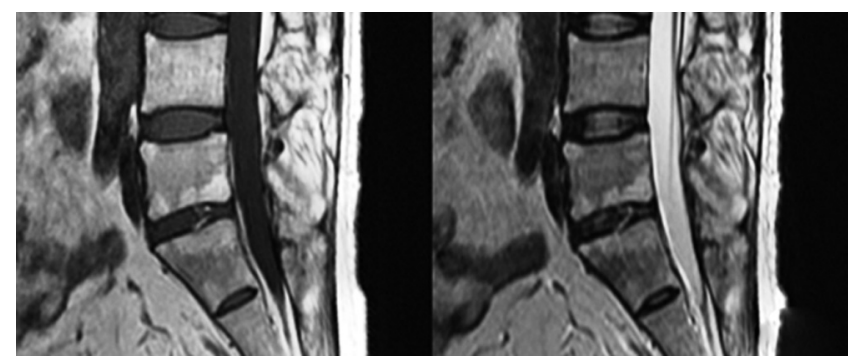

Fig. $2 b$

MR scans showing a) florid inflammatory Andersson lesions at T7-8 with acute inflammation (left, $\mathrm{T}_{2}$-weighted; right, $\mathrm{T}_{1}$-weighted) and $\mathrm{b}$ ) an old inflammatory Andersson lesion at L5-S1 with reactive fatty degeneration of the bone marrow (left, $\mathrm{T}_{2}$-weighted; right, $\mathrm{T}_{1}$-weighted).

Table II. Site of the nine

traumatic Andersson lesions

\begin{tabular}{ll}
\hline Level & Cases $(\mathbf{n}, \mathbf{\%})$ \\
\hline T10-11 & $2(22.2)$ \\
T11-12 & $1(11.1)$ \\
T12-L1 & $4(44.4)$ \\
L1-2 & $2(22.2)$ \\
\hline
\end{tabular}

$26.8^{\circ}\left(12^{\circ}\right.$ to $\left.39.5^{\circ}\right)$ and a mean acute scoliosis of $9.3^{\circ}\left(4^{\circ}\right.$ to $15^{\circ}$ ), the lesions being at the apex of the curve (Fig. 3). On MRI, lesions with low signal intensity were seen on the $\mathrm{T}_{1}$-weighted images and lesions with irregular signals on the 


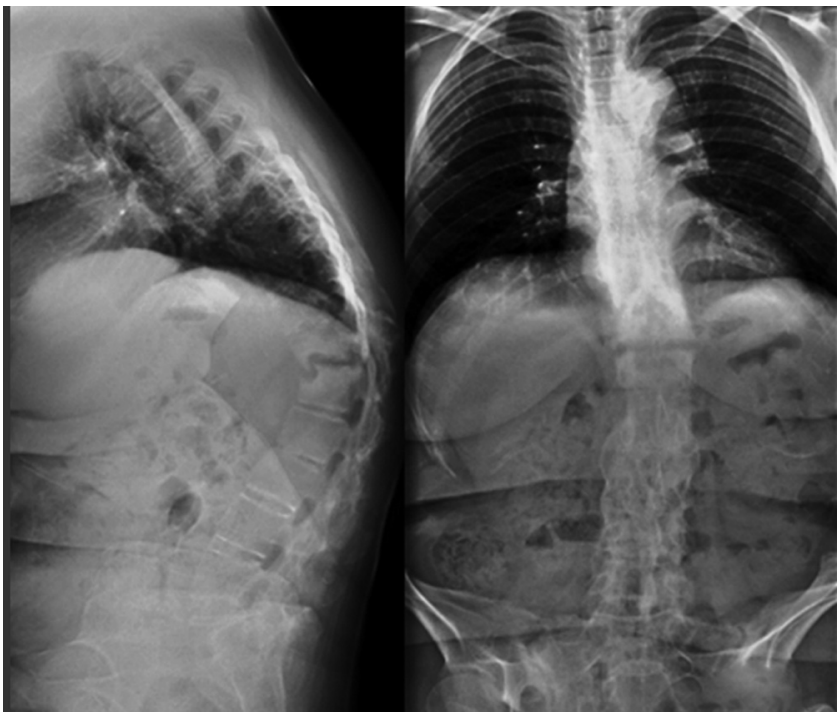

Fig. 3

Radiographs showing acute kyphotic (left) and scoliotic (right) deformity in a case of traumatic Andersson lesion at T12-L1.

$\mathrm{T}_{2}$-weighted images (Fig. 4). Traumatic lesions occurred when bony ankylosis was relatively well developed compared to the inflammatory lesions. The mean mSASSS in these nine patients with traumatic lesions was higher than that in the patients with inflammatory lesions (34.14 (12 to 66) versus 16.05 (10 to 25 ), respectively).

Clinical findings. There was a difference in the mean duration of disease for the two types of lesion: 9.4 years (1.4 to 21) for the inflammatorylesions and 17.2 years ( 4.3 to 34 ) for traumatic lesions. Of the 33 patients with Andersson lesions, 11 (seven with an inflammatory lesion and four with a traumatic lesion) complained of pain at the site. However, the nature of the pain was mechanical (i.e., related to movement) in the case of traumatic lesions and inflammatory (i.e., constant pain disturbing sleep, stiffness after periods of immobility) in the case of inflammatory lesions.

A previous history of trauma was confirmed as the cause of the lesion in four of the nine patients with a traumatic lesion. All the injuries were minor (three were falls and one a traffic accident) and had occurred at a mean of 51 months (3 to 84 ) previously. None of the patients had any neurological abnormality.

Findings of blood and histology. The mean ESR and CRP were $42.84 \mathrm{~mm} / \mathrm{hr}$ (10 to 146 ) and $3.09 \mathrm{mg} / \mathrm{l}(0.13$ to $12.10)$ in the inflammatory lesions, respectively, and $36.79 \mathrm{~mm} / \mathrm{hr}$ (4 to 76$)$ and $2.93 \mathrm{mg} / \mathrm{l}(0.47$ to 12.50$)$ in the traumatic lesions, respectively. Human leucocyte antigen (HLA) B-27 was present in 21 of the 24 patients with an inflammatory lesion and in eight of the nine patients with a traumatic lesion. Biopsies were obtained from five of the patients with a traumatic lesion during the course of anterior interbody fusion. The biopsy material showed evidence of bony fragments, fibrosis and chondrodysplasia in addition to chronic inflammation.
Treatment and changes during follow-up. Patients were initially treated with non-steroidal anti-inflammatory drugs (NSAIDs). If their symptoms failed to improve, we used an anti-tumour necrosis factor (TNF) agent, especially for those with inflammatory lesions. This had an excellent effect on their back pain. In patients with an inflammatory lesion, the inflammation was suppressed and central bony ankylosis had occurred at mid- to long-term follow-up (Fig. 5a). In some cases peripheral bony ankylosis occurred around the annulus fibrosus, giving the appearance of a bamboo spine (Fig. 5b). Unlike those with inflammatory lesions, patients with traumatic lesions showed signs of spinal instability because of severe damage to the vertebral bodies and an ununited posterior column. These appearances improved with conservative treatment in three patients (Fig. 6) and after corrective surgery in five of the other six.

For these, we carried out an anterior interbody fusion around the lesion, a Smith-Petersen osteotomy ${ }^{15}$ in an asymmetric V-shape at the level of the lesion, and a pedicle subtraction osteotomy of the lumbar spine to correct the sagittal imbalance (Fig. 7). We achieved a mean correction of the kyphotic deformity of $8.9^{\circ}\left(8^{\circ}\right.$ to $\left.10^{\circ}\right)$ and a mean correction of the scoliotic deformity of $8.4^{\circ}\left(4^{\circ}\right.$ to $\left.14^{\circ}\right)$. The thoracic kyphosis was corrected from a mean of $49.1^{\circ}\left(30^{\circ}\right.$ to $\left.69^{\circ}\right)$ to $42.3^{\circ}\left(25^{\circ}\right.$ to $\left.57^{\circ}\right)$, and the mean lumbar lordosis was corrected from $-8.8^{\circ}\left(-12^{\circ}\right.$ to $\left.-5^{\circ}\right)$ to $-39.0^{\circ}\left(-51^{\circ}\right.$ to $\left.-32^{\circ}\right)$. The list (the distance between a perpendicular line drawn from the centre of the $\mathrm{C} 7$ body to the posterosuperior corner of S1) was also reduced from a mean of $8.6 \mathrm{~cm} \mathrm{(2.2} \mathrm{to} 15.4)$ to a mean of $3.8 \mathrm{~cm}$ (0 to 6.5$)$.

\section{Discussion}

The prevalence of destructive discovertebral lesions in ankylosing spondylitis ranges from 1 to 28 per 100 cases. ${ }^{2,3,16}$ In this study, the overall prevalence of Andersson lesions was $5.3 \%$ (3.9\% inflammatory and $1.4 \%$ traumatic). The sensitivity of MRI for inflammatory lesions is known to be higher than that of plain radiography. ${ }^{11,12}$ Since only $6.3 \%$ of our patients were investigated by MRI, the real prevalence was almost certainly higher.

Infection, inflammation and pseudarthrosis have all been considered to be causes of Andersson lesions. The associated pain and radiological features are reported to have improved after treatment with NSAIDs and anti-TNF agents. ${ }^{5,12,17}$ However, there have also been reports that surgical intervention, in the form of interbody fusion, is needed to relieve the pain caused by instability, kyphotic deformities and neurological disorders. ${ }^{9}, 18,19$

In early studies the lesions were thought to be caused by infection because of their radiological similarity to an infective spondylodiscitis, ${ }^{20}$ but bacteriological and other investigations failed to support this view. ${ }^{3,19,21}$ Today, the lesions are explained in terms of inflammatory and traumatic causes. Support for an inflammatory cause is based on the absence of a previous history of trauma, features of chronic 


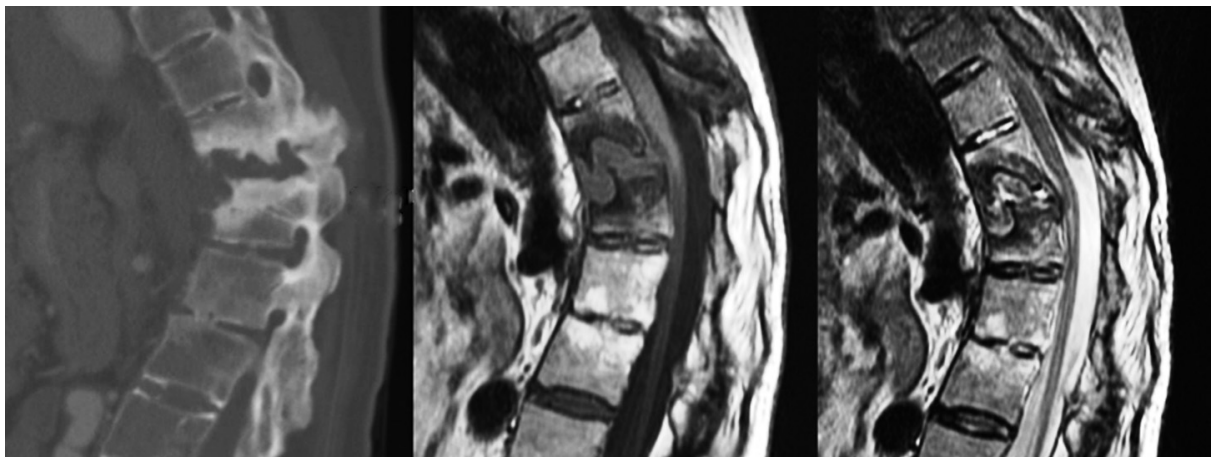

Fig. 4

CT scan (left) and MR scans (centre, $T_{1}$-weighted; right, $T_{2}$-weighted) showing nonunion of a posterior column fracture at T12 in a case of traumatic Andersson lesion. Low signal intensities are seen in the $T_{1}$-weighted image and irregular signals in the $\mathrm{T}_{2}$-weighted image.

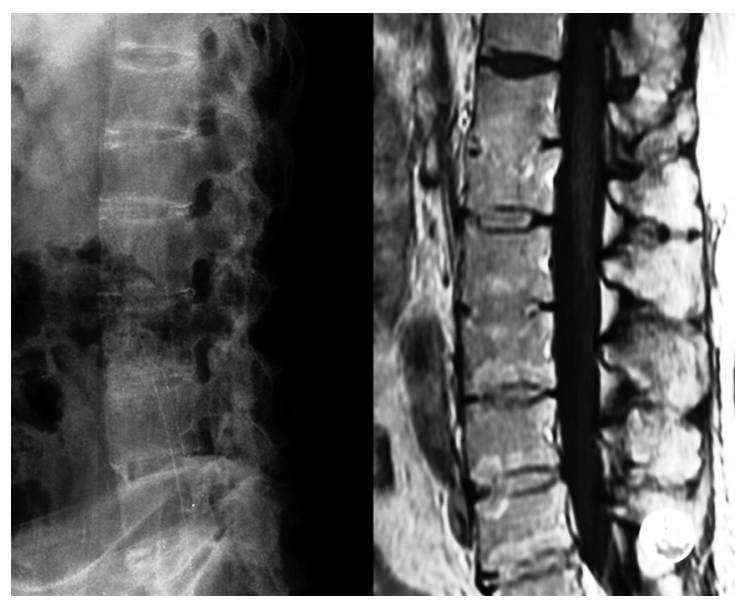

Fig. $5 a$

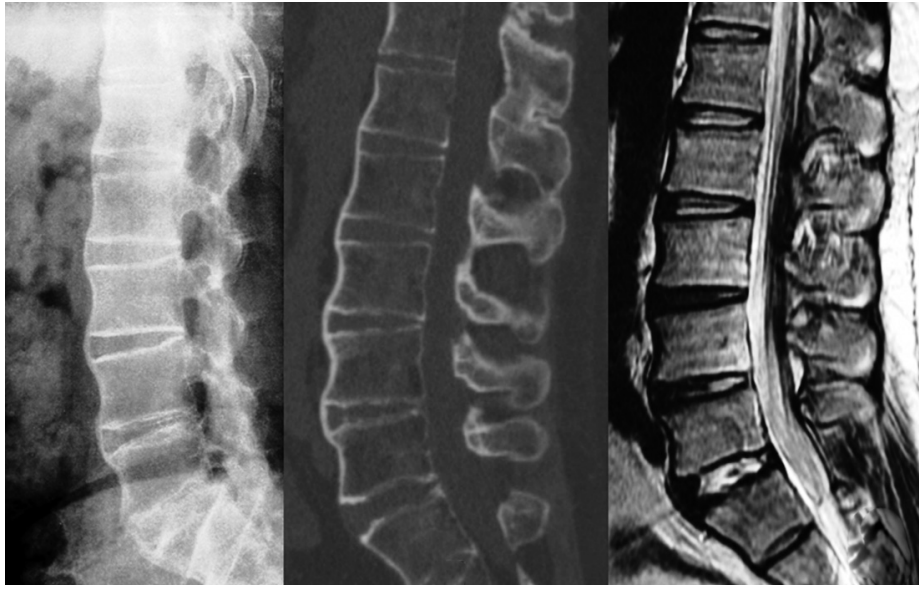

Fig. $5 b$

Figure $5 \mathrm{a}$ - radiograph (left) and $\mathrm{T}_{2}$-weighted MR scan (right) showing central (transdiscal) bony ankylosis at the levels T12-L1 and L2-3 in a case of inflammatory Andersson lesion. Figure $5 \mathrm{~b}$ - radiograph (left), CT scan (centre) and $\mathrm{T}_{2}$-weighted MR scan (right) showing peripheral (prediscal) bony ankylosis at the whole lumbar spine.

non-bacterial inflammation on pathological examination, and improvement of the patient's pain and radiological features with anti-inflammatory drugs. ${ }^{5,6,12,17}$ A traumatic cause is predicated on the appearance of a nonunion after an acute traumatic or insufficiency fracture, and occurs frequently among those with a previous history of trauma and/or a heavy job, and is confirmed histologically by the presence of fibrosis and chondrodysplasia. ${ }^{8}$

In this study, four of the nine patients with a traumatic lesion had a previous history of trauma, which was assumed to be the cause of the lesions, whereas patients with inflammatory lesions had no such history. Also, in five patients with traumatic lesions bony fragments, fibrosis and chondrodysplasia were found in the tissue removed during anterior interbody fusion.

Narrowing of the disc space and abnormal radiodensity of the vertebral body have been reported as being characteristic features of inflammatory lesions, ${ }^{11,12,22}$ and this was confirmed by our study. Many of the features of inflammatory lesions are similar to those of Romanus lesions, ${ }^{23}$ which are early inflammatory lesions located in the anterior corners of the vertebral bodies; apart from the difference in location, they are radiologically identical and, in particular, give the same changes in signal intensity on MRI. By contrast, widespread destruction of the vertebral body and discs, and nonunion of the resultant posterior column fractures, have been reported as distinctive features of traumatic lesions. ${ }^{10-12}$ These characteristics were also confirmed by this study.

Owing to the inflammatory nature of the disease more than half of all patients with ankylosing spondylitis develop osteoporosis. Because of this and the reduced spinal flexibility due to bony ankylosis, these patients are thought to be more susceptible to spinal fractures. ${ }^{10}$ This view was confirmed in this study, as patients with a traumatic lesion had a higher mSASSS than those with 


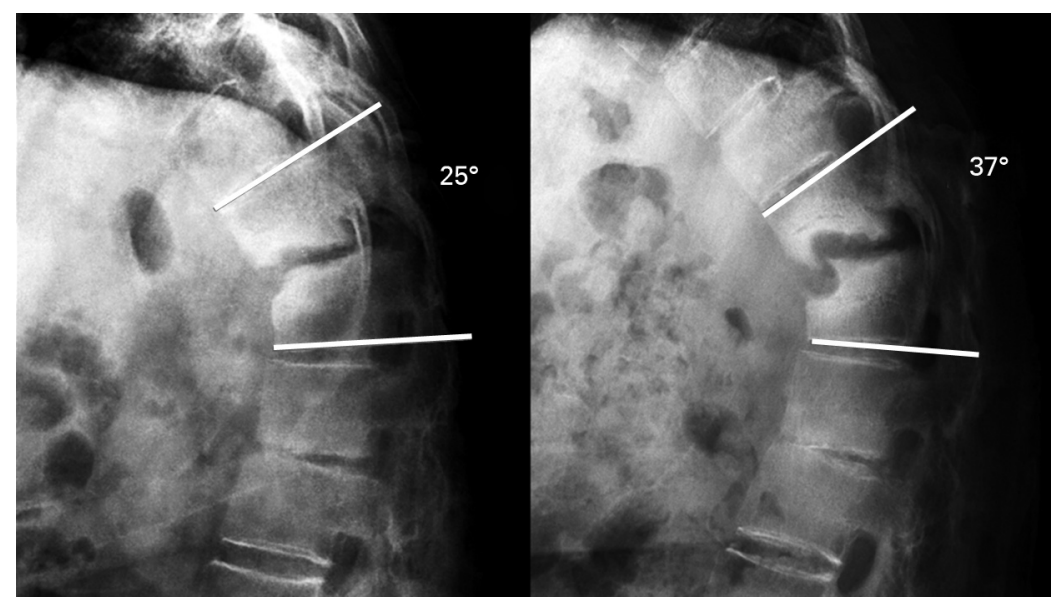

Fig. 6

Radiographs of a 46 -year-old female showing a kyphotic deformity of $25^{\circ}$ (left) and the progression of the deformity of $37^{\circ}$ at four years' follow-up (right) in a case of traumatic Andersson lesion.

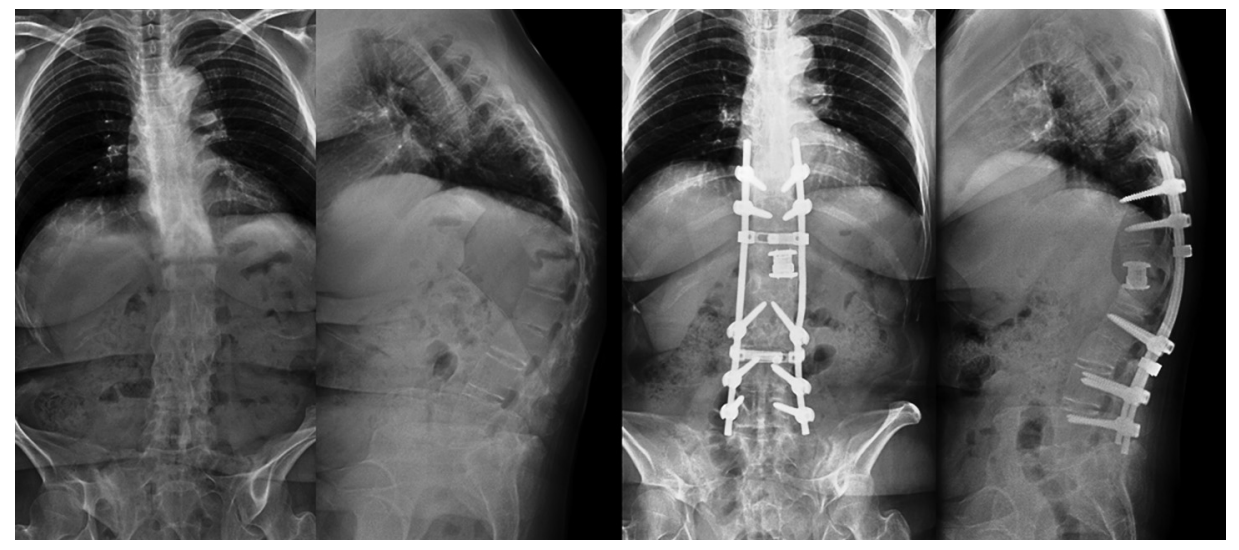

Fig. 7

Anteroposterior and lateral radiographs of a 46-year-old female patient with a traumatic Andersson lesion at T12-L1 pre-operatively (left), showing scoliotic and kyphotic deformities, and post-operatively at one year (right), after anterior interbody fusion with cage, asymmetric Smith-Petersen osteotomy and L3 pedicle subtraction osteotomy, showing correction of the deformity and the sagittal imbalance (right).

inflammatory lesions. It is also reported that the stresses around the thoracolumbar junction are radically increased when these lesions are accompanied by a kyphotic deformity. ${ }^{10}$ Among the nine cases of traumatic lesions in this study, increased stress was generated at the thoracolumbar junction in six cases, between T10 and T11 in two further cases, and between L1 and L2 in the remaining case. Once a fracture occurs in an ankylosed spine, bony union is hampered by constant movement at the solitary mobile segment between two long fused segments. Consequently, traumatic lesions tend to have radiological features that differ from those of inflammatory lesions, and resemble hypertrophic pseudarthrosislike nonunions in a long bone with nonunion and delayed union of the associated posterior column fracture. This was true of all nine patients in our study.

Pain and the evolution of deformity due to instability have previously been reported. ${ }^{9}$ In this study, three of the four patients with a traumatic lesion who complained of pain were found to have a radiologically unstable spine.

In summary, in this study we subdivided the discovertebral (Andersson) lesions of ankylosing spondylitis into inflammatory and traumatic types and confirmed the clinical and radiological differences between the two. The fact that many previous studies used a single term for two quite different pathological phenomena has caused a great deal of confusion. We believe our findings permit a clear distinction to be made between the two types. This should 
clarify the clinical picture and provide the appropriate criteria for determining treatment. Put simply, we suggest that anti-TNF agents be used to treat the pain of inflammatory lesions, whereas operative treatment be used to alleviate the pain, deformity and instability of traumatic lesions.

No benefits in any form have been received or will be received from a commercial party related directly or indirectly to the subject of this article.

\section{References}

1. Andersson 0. Rontgenbilden vid spondylarthritis ankylopoetica. Nord Med 1937;14:2000-2 (in Swedish).

2. Chan FL, Ho EK, Fang D, et al. Spinal pseudarthrosis in ankylosing spondylitis. Acta Radiol 1987;28:383-8.

3. Fang D, Leong JC, Ho EK, Chan FL, Chow SP. Spinal pseudoarthrosis in ankylosing spondylitis: clinicopathological correlation and the results of anterior spinal fusion. $J$ Bone Joint Surg [Br] 1988;70-B:443-7.

4. Frank $\mathbf{P}$, Gleeson JA. Destructive vertebral lesions in ankylosing spondylitis. $\mathrm{Br} \mathrm{J}$ Radiol 1975;48:755-8.

5. Rasker JJ, Prevo RL, Lanting PJ. Spondylodiscitis in ankylosing spondylitis, inflammation or trauma?: a description of six cases. Scand J Rheumato/1996;25:52-7.

6. Little H, Urowitz MB, Smythe HA, Rosen PS. Asymptomatic spondylodiscitis: an unusual feature of ankylosing spondylitis. Arthritis Rheum 1974;17:487-93.

7. Hansen ST Jr, Taylor TK, Honet JC, Lewis FR. Fracture-dislocations of the ankylosed thoracic spine in rheumatoid spondylitis: anklylosing spondylitis, MarieStrümpell disease. J Trauma 1967;7:827-37.

8. Kanefield DG, Mullins BP, Freehafer AA, et al. Destructive lesions of the spine in rheumatoid ankylosing spondylitis. J Bone Joint Surg [Am] 1969;51-A:1369-75.

9. Kim KT, Suk KS, Lee SH, Bae SC. The treatment of spinal pseudarthrosis in ankylosing spondylitis. Spine 2006;31:1351-7.

10. Bron JL, de Vries MK, Snieders MN, van der Horst-Bruinsma IE, van Royen BJ. Discovertebral (Andersson) lesions of the spine in ankylosing spondylitis revisited. Clin Rheumatol 2009:28:883-92.
11. Hermann KG, Bollow M. Magnetic resonance imaging of the axial skeleton in rheu matoid disease. Best Prac Res Clin Rheumatol 2004;18:881-907.

12. Hermann KG, Althoff CE, Schneider U, et al. Spinal changes in patients with spondyloarthritis: comparison of MR imaging and radiographic appearances. Radiographics 2005:25:559-70.

13. van der Linden S, Valkenburg HA, Cats A. Evaluation of diagnostic criteria for ankylosing spondylitis: a proposal for modification of the New York criteria. Arthritis Rheum 1984;27:361-8.

14. Creemers MC, Franssen MJ, van't Hof MA, et al. Assessment of outcome in ankylosing spondylitis: an extended radiographic scoring system. Ann Rheum Dis 2005;64:127-9.

15. Gill JB, Levin A, Burd T, Longley M. Corrective osteotomies in spine surgery. Bone Joint Surg [Am] 2008;90-A:2509-20.

16. Kabasakal Y, Garrett SL, Calin A. The epidemiology of spondylodiscitis in ankylosing spondylitis: a controlled study. Br J Rheumatol 1996;35:660-3.

17. Sieper J, Baraliakos $\mathbf{X}$, Listing $\mathbf{J}$, et al. Persistent reduction of spinal inflammation as assessed by magnetic resonance imaging in patients with ankylosing spondylitis after 2 years of treatment with the anti-tumour necrosis factor agent infliximab. Rheumatology 2005;44:1525-30.

18. Chang KW, Tu MY, Huang HH, et al. Posterior correction and fixation without anterior fusion for pseudarthrosis with kyphotic deformity in ankylosing spondylitis. Spine 2006;31:E408-13

19. Van Royen BJ, Kastelijns RC, Noske DP, Oner FC, Smit TH. Transpedicular wedge resection osteotomy for the treatment of a kyphotic Andersson lesion complicating ankylosing spondylitis. Eur Spine J 2006;15:246-52.

20. Lohr KM, Barthelemy CR, Schwab JP, Haasler GB. Septic spondylodiscitis in ankylosing spondylitis. J Rheumatol 1987;14:616-20.

21. Nikolaisen C, Nossent H. Early histology in ankylosing spondylitis related spondy lodiscitis supports its inflammatory origin. Scand J Rheumatol 2005;34:396-8.

22. Dihlmann W, Delling G. Disco-vertebral destructive lesions (so-called Andersson lesions) associated with ankylosing spondylitis. Skeletal Radiol 1978;3:10-16.

23. Romanus R, Yden S. Destructive and ossifying spondylitic changes in rheumatoid ankylosing spondylitis (pelvo-spondylitis ossificans). Acta Orthop Scand 1952;22:88 